\title{
Stimulated Raman Microscopy Implemented by Three Femtosecond Laser Sources
}

\author{
Rajeev Ranjan ${ }^{1,2 *}$, Maria Antonietta Ferrara ${ }^{2}$, Luigi Sirleto ${ }^{2}$ \\ ${ }^{1}$ CHT @Erzelli, Nanoscopy, Istituto Italiano Di Tecnologia, Genova, Italy \\ ${ }^{2}$ National Research Council (CNR), Institute of Applied Sciences and Intelligent Systems, Napoli, Italy, Napoli, Italy
}

\begin{abstract}
In this work, the implementation of a femtosecond Stimulated Raman Scattering microscope, equipped with three femtosecond laser sources: a Titanium-Sapphire (Ti:Sa), an optical parametric oscillator (OPO), and a second harmonic generator (SHG); is presented. Our microscope is designed so that it can cover all the regions of Raman spectra, taking advantage of two possible laser combinations. The first, Ti:Sa and OPO laser beams, which cover the $\mathrm{C}-\mathrm{H}$ region $\left(>2800 \mathrm{~cm}^{-1}\right)$ in stimulated Raman gain (SRG) modality, whereas the second, Ti:Sa and SHG laser beams, covering the C-H region and the fingerprint region in stimulated Raman losses (SRL) modality. The successful realization of the microscope is demonstrated, reporting images of polystyrene beads using both SRL and SRG modalities.
\end{abstract}

\section{Introduction}

In spite of the fact that the fluorescence microscopy represents the fundamental pillars for biological imaging, it shows significant limitations: (i) some molecules and/or biological structures cannot be labeled without changing their biological function, such as lipids; (ii) many labels are cytotoxic, which leads to interference with the biological functionality. For these reasons, label-free imaging, obtained by microscopy techniques, is highly desirable, affording high chemical selectivity of unlabeled living cells.

In the last decades, nonlinear optics microscopy has become an attractive tool in both biological and biomedical research to overcome limitations due to fluorescence microscopy. By exploiting nonlinear optical effects, novel microscopy techniques have been developed, which afford high chemical selectivity of unlabeled living cells and implement real-time threedimensional imaging with high spatial resolution and sensitivity. In multimodal imaging, various nonlinear optics microscopies (CARS, SRS, SHG, THG, and SFG) are combined in a single microscope to image cells or tissue by simultaneous image acquisition. The significant advantage is that the same excitation source can be used for all the nonlinear optics microscopy techniques in this combination of methods $[1,2]$.

Among novel label-free nonlinear microscopy techniques, Stimulated Raman scattering (SRS) techniques play an essential role. Over the past ten years, SRS has been investigated in nanophotonic [3-6] as well as in biophotonics as an analytical, label-free, noninvasive technique with unique cellular and tissue imaging capabilities [7-10]. SRS technique is sensitive to the same molecular vibrations, probed in spontaneous Raman spectroscopy, but it exhibits a nonlinear dependence on the incoming light fields and produces coherent radiation. In SRS microscopy, two collinear laser beams, a high-power pump laser and a low-power Stokes laser with different frequencies $\left(\omega_{L}>\omega_{S}\right)$ are focused on a sample to generate an SRS signal. When their difference resonantly excites the vibrational frequency of the molecular bonds of interest, energy is transferred from the pump beam to the probe beam, observable in an increase in probe signal intensity (Stimulated Raman Gain, SRG) and a decrease of the pump signal intensity (Stimulated Raman Loss, SRL) $[3,10]$.

In bio-photonics applications, label-free SRS microscopy allows the image of several molecular species, targeting their intrinsic chemical bonds at the congested cellular fingerprint region $\left(<1800 \mathrm{~cm}^{-1}\right)$ or at the high-frequency $\mathrm{C}-\mathrm{H}$ region $\left(>2800 \mathrm{~cm}^{-1}\right)$. Moreover, recently, a bioorthogonal chemical imaging platform has emerged by coupling SRS microscopy with tiny and Raman-active vibrational probes (e.g., alkynes and stable isotopes). Spectroscopically, these Raman active vibration probes exhibit Raman peaks at the cell-silent region where no other peaks from endogenous molecules exist, demonstrating sensitivity, specificity, and biocompatibility for imaging small biomolecules [11, 14].

In the reference [15], nonlinear vibrational bioimaging was demonstrated by taking advantage of femtosecond stimulated Raman loss. It is worth noting that a 12 times more significant SRL loss signal by femtosecond pulse

\footnotetext{
*Corresponding author: rajeev.ranjan@iit.it
} 
excitation was measured with respect to picosecond pulse. Definitely, due to the low sensitivity of Ramanactive vibrational probes, femtosecond stimulated Raman microscopy appears as an unavoidable perspective. In addition, interesting biological applications based on femtosecond SRS microscopy have been reported in the literature, extending in the silent region and taking advantage of bio-orthogonal chemical imaging platform [16].

We note that typical and widespread commercial laser sources combinations, such as Chameleon compact OPO (Coherent, Inc.) and Ti:Sa, although tailored for multimodal imaging, their minimum Raman shift is 2500 $\mathrm{cm}^{-1}$. This means that by adopting this laser combination, only the $\mathrm{CH}-\mathrm{OH}$ region of Raman spectra can be explored, while the silent and fingerprint regions are out of emission range [17-22]. Therefore, they cannot accomplish the demand of biorthogonal platforms based on femtosecond stimulated Raman microscopy.

\section{Method and Results}

In this work, the implementation of a femtosecond SRS microscope, covering all the regions of Raman spectra, is described. The microscope is equipped with three femtosecond laser sources: a Ti:Sapphire (Ti:Sa), a synchronized optical parametric oscillator (SOPO), and a frequency converter for ultrafast lasers, i.e., a second harmonic generator ( $\mathrm{SHG}$ ) optimized for the SOPO, which allows doubling the OPO energy radiation.

This SRS microscope is used to take advantage of two laser combinations. Ti:Sa and OPO laser combination cover the $\mathrm{C}-\mathrm{H}$ region in SRG modality[17-22]. The latter Ti:Sa and SHG laser combination provide the extension of the microscope to the silent region $(<1800$ $\mathrm{cm}-1)$ and fingerprint region (1800-2800 $\mathrm{cm}-1)$ in the SRL modality [23].

In our experimental setup, a flip mirror (FM) placed after the OPO exit is used in order to switch the Ti:Sa-OPO and Ti:Sa-SHG laser combinations. When the FM is inserted in the optical path, OPO radiation is coupled into SHG, so Ti:Sa-SHG laser combination becomes available, whereas when the FM is flipped, the OPO can go on undisturbed, and Ti:Sa-OPO laser combination becomes available (see Figure 1). Considering the Ti:Sa laser excitation within the wavelength range of 740-880 $\mathrm{nm}$, the corresponding OPO emission range is 1000 $1600 \mathrm{~nm}$, while the SHG emission range is $500-800 \mathrm{~nm}$. When the Ti:Sa is used as pump at $830 \mathrm{~nm}$, the largest range of Raman shift is obtained. Indeed, in this case, all the regions of Raman spectra can be covered (from 451 $\mathrm{cm}^{-1}$ to $3195 \mathrm{~cm}^{-1}$ ) using the Ti:Sa-SHG laser combination[23].

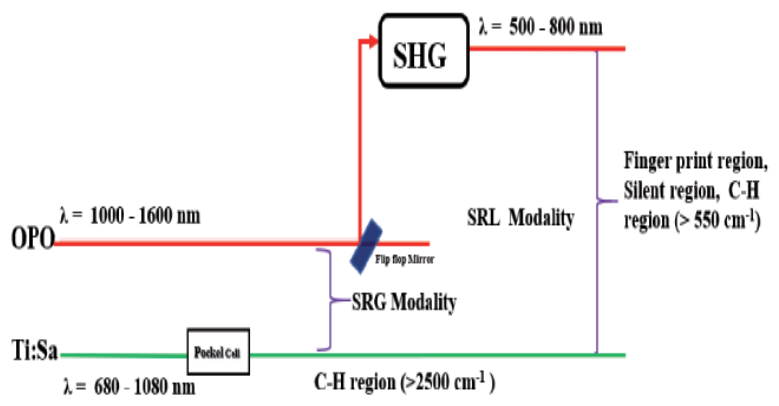

Figure 1. Generation system with combination Ti:SaOPO (SRG modality) and Ti:Sa-SHG (SRL modality).

In order to demonstrate its successful realization SRG and SRL images of polystyrene beads with a diameter of $3 \mu \mathrm{m}$ are reported in figures 2 and 3, respectively, along with transmission image (TI). All the images are single recordings of $512 \mathrm{px} \times 512 \mathrm{px}$, and their acquisition

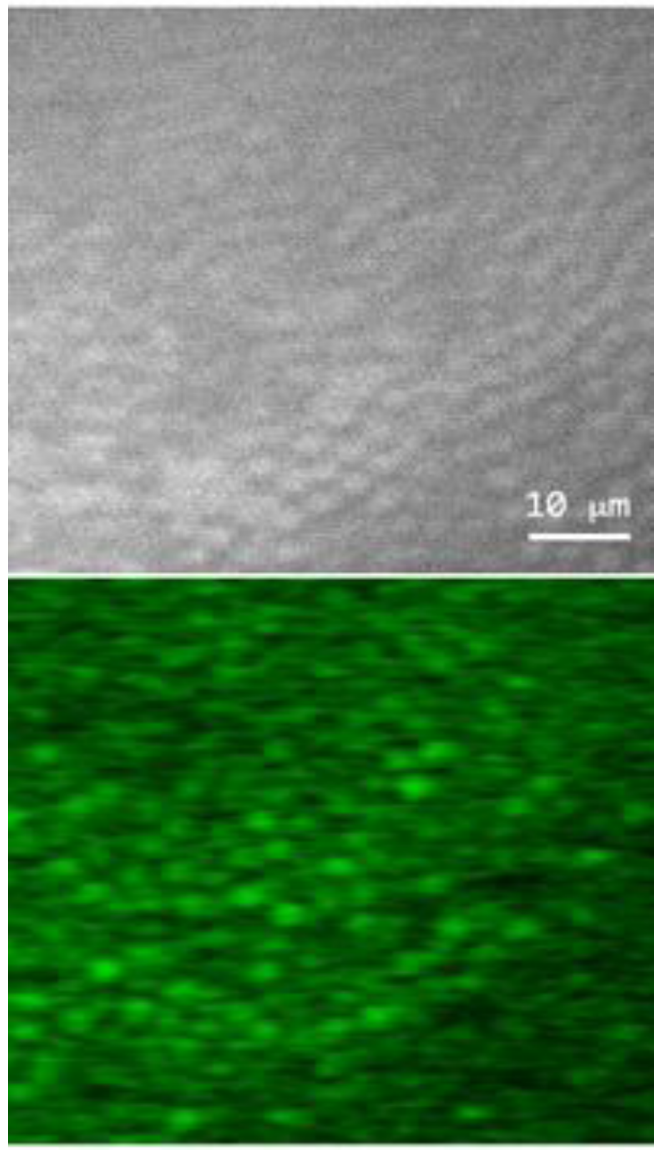

Figure 2. On the top transmission image, the bottom SRG image of polystyrene beads sample with a diameter of $3 \mu \mathrm{m}$.

time is $16 \mathrm{~s}$. The Lock-in amplifier (LIA) time constant was set to $100 \mathrm{~s}$ with an $18 \mathrm{~dB} /$ oct slope and a sensitivity of $10 \mathrm{~V}$. For SRG images, the pump signal generated by Ti:Sa was set at $808 \mathrm{~nm}$, while the probe signal generated by OPO was set at $1072 \mathrm{~nm}$. For both of them, the average focused power was about $10 \mathrm{~mW}$. For SRL 
imaging, the pump signal generated by the SHG unit was set at $650 \mathrm{~nm}$, while the probe signal generated by Ti:Sa was set at $811 \mathrm{~nm}$, for Ti:Sa, the average focused power was $8 \mathrm{~mW}$, while for $\mathrm{SHG}$, the average focused power was of $6 \mathrm{~mW}$.

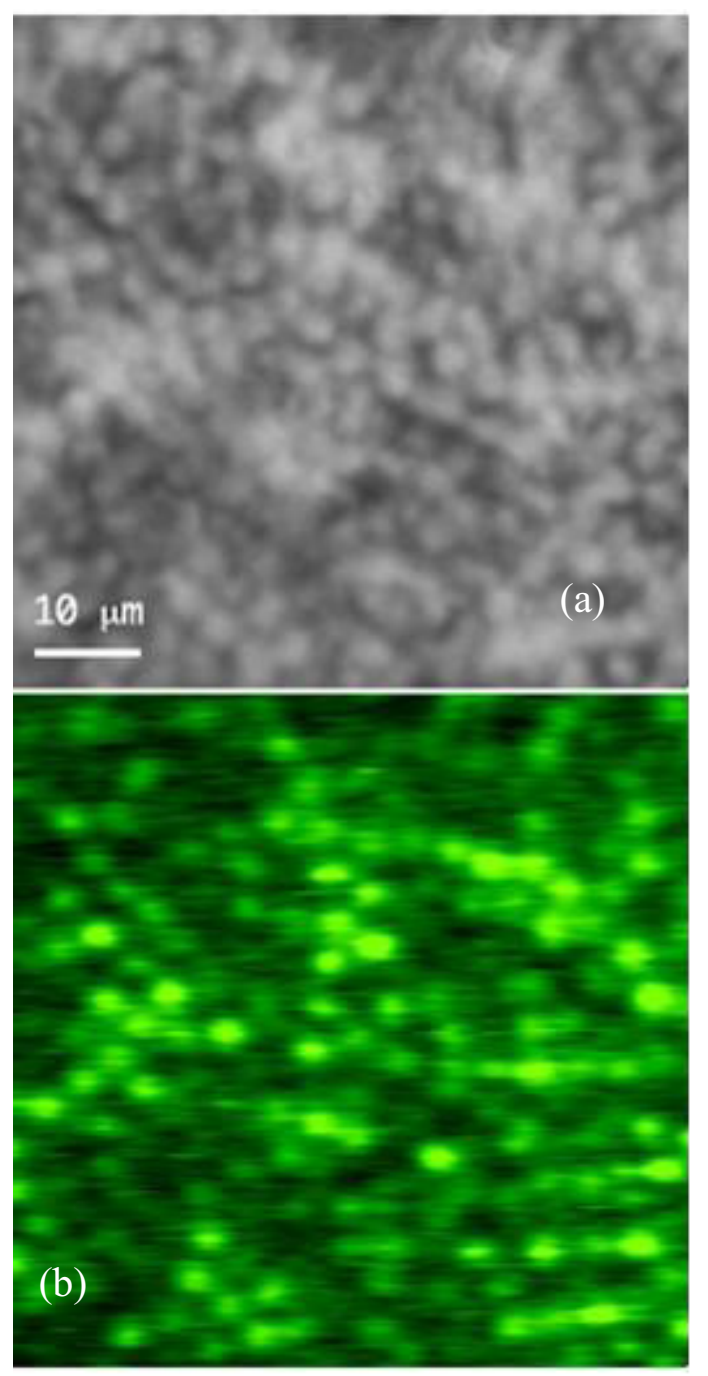

Figure 3 (a) Transmission image and (b) SRL image of a polystyrene beads sample with a diameter of $3 \mu \mathrm{m}$.

\section{CONCLUSIONS}

In conclusions, we have demonstrated the implementation of a femtosecond stimulated Raman microscope, extending its working range in fingerprint and in silent regions. Our microscope has been designed in order to allow the coexistence of SRL and SRG optical modalities without modifying optical path, i.e. without adding, removing or moving components.

\section{ACKNOWLEDGEMENTS}

The authors would like to thank M. Indolfi and V. Tufano (ISASI-CNR) for their technical assistance.

\section{References}

1. Streets, A. M.; Li, A.; Chen, T.; Huang, Y. Imaging without fluorescence: nonlinear optical microscopy for quantitative cellular imaging. Anal. Chem. 2014, 86, 8506-8513.

2. Min, W.; Freudiger, C.W.; Lu, S.; Xie, X.S. Coherent nonlinear optical imaging: beyond fluorescence microscopy. Annu. Rev. Phys. Chem. 2011, 62, 501-530.

3. Sirleto, L.; Ferrara, M.A.; Nikitin, T.; Novikov, S.; Khriachtchev, L. Giant Raman gain in silicon nanocrystals. Nat. Commun. 2012, 3, 1-6. doi: 10.1038/ncomms2188;

4. Sirleto, L.; Vergara, A.; Ferrara, M.A. Advances in stimulated Raman scattering in nanostructures. Adv. Opt. Photon. 2017, 9, 169-217. doi: 10.1364/AOP.9.000169

5. M.A. Ferrara, L.Sirleto, G.Nicotra, C.Spinella, I.Rendina. Enhanced gain coefficient in Raman amplifier based on silicon nanocomposites. 2011 vol.9, pp.1-7. doi:10.1016/j.photonics.2010.07.007.

6. Sirleto, L.; Aronne, A.; Gioffrè, M.; Fanelli, E.; Righini, G.C.; Pernice, P.; Vergara, A. Compositional and thermal treatment effects on Raman gain and bandwidth in nanostructured silicabased glasses. Opt. Mater. 2013, 36, 408-413. http://dx.doi.org/10.1016/j.optmat.2013.10.001

7. Freudiger, C.W.; Min,W.; Saar, B.G.; Lu, S.; Holtom, G.R.; He, C.; Tsai, J.C.; Kang, J.X.; Xie, X.S. Label-Free Biomedical Imaging with High Sensitivity by Stimulated Raman Scattering Microscopy. Science 2008, 322, 1857-1861. [CrossRef] [PubMed]

8. Cheng, J.-X.; Xie, X.S. Vibrational spectroscopic imaging of living systems: An emerging platform for biology and medicine. Science 2015, 350, aaa8870. [CrossRef]

9. Camp, C.H., Jr.; Cicerone, M.T. Chemically sensitive bioimaging with coherent Raman scattering. Nat. Photon 2015, 9, 295-305. [CrossRef]

10. Zumbusch, A.; Langbein,W.; Borri, P. Nonlinear vibrational microscopy applied to lipid biology. Prog. Lipid Res. 2013, 52, 615-632. [CrossRef]

11. Wei, L.; Hu, F.; Shen, Y.; Chen, Z.; Yu, Y.; Lin, C.; Wang, M. C.; Min, W. Live-cell imaging with alkyne tagged small biomolecules by stimulated Raman Scattering. Nat. Methods, 11, 410-412 (2014).

12. Wei, Y Shen, F Xu, F Hu, JK Harrington, KL Targoff, et al.: Imaging complex protein metabolism in live organisms by stimulated Raman scattering microscopy with isotope labeling, ACS Chemical Biology, vol. 10, no. 3, pp. 901-908, 2015.

13. Fanghao $\mathrm{Hu}$, Michael R. Lamprecht, LuWei, Barclay Morrison Wei Min. Bioorthogonal chemical imaging of metabolic activities in live mammalian hippocampal tissues with stimulated Raman 
scattering. Scientific Reports | 6:39660 | DOI: 10.1038/srep39660.

14. Lu Wei, Fanghao Hu, Zhixing Chen, Yihui Shen, Luyuan Zhang, and Wei Min, Live-Cell Bioorthogonal Chemical Imaging: Stimulated Raman Scattering Microscopy of Vibrational Probes. Acc. Chem. Res.,2016,49(8), pp 1494-1502. DOI: 10.1021 acs.accounts.6b00210

15. D. Zhang, M. N. Slipchenko, and J. X. Cheng, $J$. Phys. Chem. Lett., vol. 2, no. 11, pp. 1248-1253, 2011.

16. C. Zhang, K.-C. Huang, B. Rajwa, J. Li, S. Yang, H. Lin, C.-S. Liao, G. Eakins, S. Kuang, V. Patsekin,J. P. Robinson, and J.-X. Cheng: Stimulated Raman scattering flow cytometry for label-free singleparticle analysis, Optica, vol. 4, no. 1, 2017

17. R. Ranjan, M. A. Ferrara, A. Filograna, C. Valente and L. Sirleto, "Femtosecond Stimulated Raman Microscopy: Home-built realization and a case study of Biological imaging", Journal of Instrumentation 14, P09008 (2019).

18. R. Ranjan, M. Indolfi, M. A. Ferrara, L. Sirleto, "Implementation of a Nonlinear Microscope Based on Stimulated Raman Scattering", J. Vis. Exp. 149, e59614 (2019).

19. M. A. Ferrara, A. Filograna, R. Ranjan, D. Corda, C. Valente, L. Sirleto, "Three-dimensional label-free imaging throughout adipocyte differentiation by stimulated Raman microscopy", PLoS ONE 14, e0216811 (2019).

20. A. D'Arco, M. A. Ferrara, M. Indolfi, V. Tufano and L. Sirleto, "Label-free imaging of small lipid droplets by femtosecond-stimulated Raman scattering microscopy", Journal of Nonlinear Optical Physics \& Materials 26, 1750052 (2017).

21. A. D'Arco, N. Brancati, M. A. Ferrara, M. Indolfi, M. Frucci, L. Sirleto, "Subcellular chemical and morphological analysis by stimulated Raman scattering microscopy and image analysis techniques", Biomedical Optics Express 7, p. 18531864 (2016).

22. Sirleto, L.; Ranjan, R.; Ferrara, M.A., Analysis of Pulses Bandwidth and Spectral Resolution in Femtosecond Stimulated Raman Scattering Microscopy. Appl. Sci. 2021, 11, 3903.

23. R. Ranjan, A. D'Arco, M. A. Ferrara, M. Indolfi, M. Larobina and L. Sirleto, "Integration of stimulated Raman gain and stimulated Raman losses detection modes in a single nonlinear microscope", Optics Express 26, pp. 26317-26326 (2018). 